\title{
Thermodynamics and Its Basic Processes via Art
}

\author{
Abraham Tamir* \\ Department of Chemical Engineering, Israel \\ *Corresponding author: Abraham Tamir, Department of Chemical Engineering, Israel,
}

Submission: 監 August 21, 2017; Published: 想 September 18, 2017

\section{Opinion}

Thermodynamics is the basis of all subjects in chemical engineering. It is defined $[1,2]$ as the area of science that includes the relationship between heat and other kinds of energy. More specifically, it is the study of energy transfer in systems in which there are changes in temperature, pressure and volume at the macroscopic scale where energy is defined as the capacity of a physical system to perform work [3]. Energy comes in different forms such as heat, light, mechanical, electrical, chemical, and nuclear energy and is used to do everything we do. Sources of energy are coals, natural gas, petroleum, wind power, geothermal energy, solar energy and nuclear energy. The history of thermodynamics [4] started with G. Galilei (1597) who introduced the concept of temperature and invented one of the first thermometers. It was G. Black (1770) who was the first to use the term "Thermodynamics" where Thompson (1854) introduced this term into conventional use. In $1772 \mathrm{G}$. Wilke introduced the unit of measuring the amount of heat, a calorie. With expansion of the knowledge in this field, thermodynamics started to include specialty areas, and accordingly the subject of thermodynamics as a science has became formulated in various topics such as: classical thermodynamics, statistical thermodynamics, non-equilibrium thermodynamics, and the axiomatic method of thermodynamics, chemical thermodynamics and engineering thermodynamics. Arnold Sommerfeld [5], a German physicist, made some amusing remark about thermodynamics. He claimed that the first time you go through thermodynamics; you don't understand it at all. The second time you go through it, you think you understand it, except for one or two small points. The third time you go through it, you know you don't understand it, but by that time you are so used to it that it doesn't bother you anymore. Thermodynamics is based on four laws that put constraints on how energy can be used in the universe. The laws touch on almost every aspect of scientific inquiry in some way and will be elaborated in future articles. It is interesting to note that the zeroth law was defined in the $20^{\text {th }}$ century where the other three in the $19^{\text {th }}$ century. Figure $1,[6]$ shows an artistic demonstration of thermodynamics by the artwork "The Sower" painted by Vincent van Gogh (1853-1890) a Dutch Post-Impressionist artist. The two basic elements in the painting are sun and man. The first one demonstrates heat transfer by the sun and the second element demonstrates a workingman where the combination of the two demonstrates what thermodynamics is, namely "the science that deals with heat and work, and the transformation of one into the other" [7]. The latter definition summarizes also the aforementioned definitions.

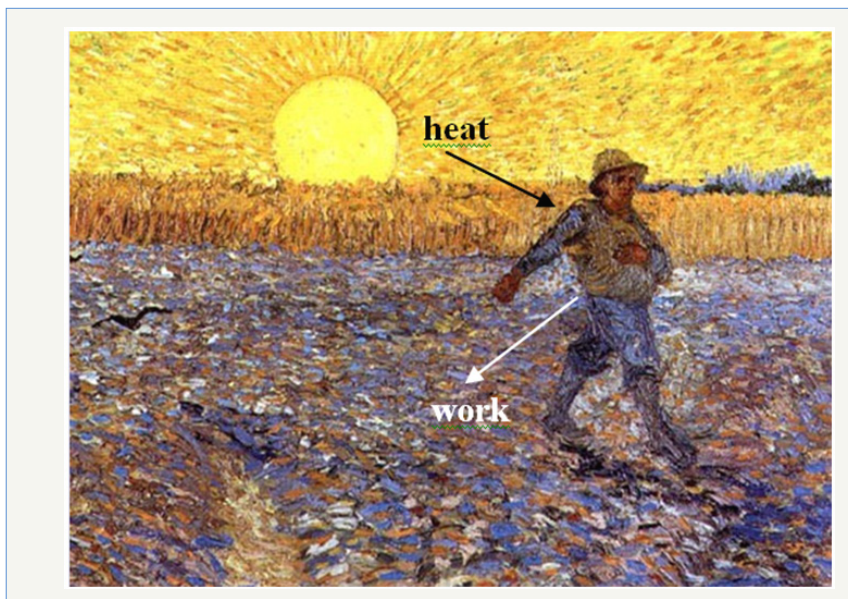

Figure 1: Thermodynamics - the transformation of heat to work.

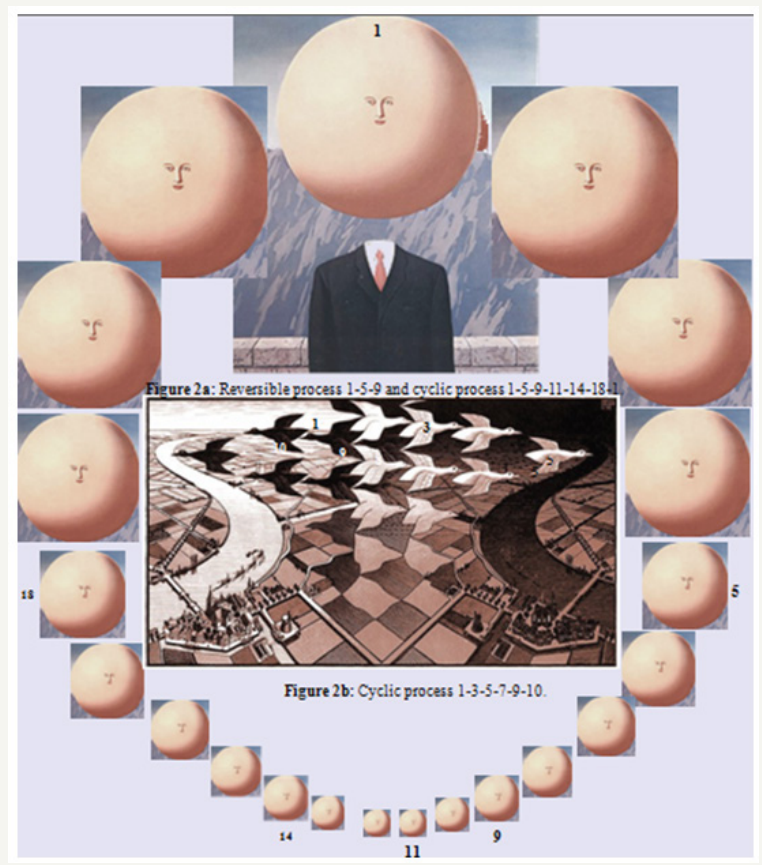

Figure 2a $\mathbf{8} \mathbf{2 b}$ : Reversible process 1-5-9 and cyclic process 1-5-9-11-14-18-1, cyclic process 1-3-5-7-9-10. 


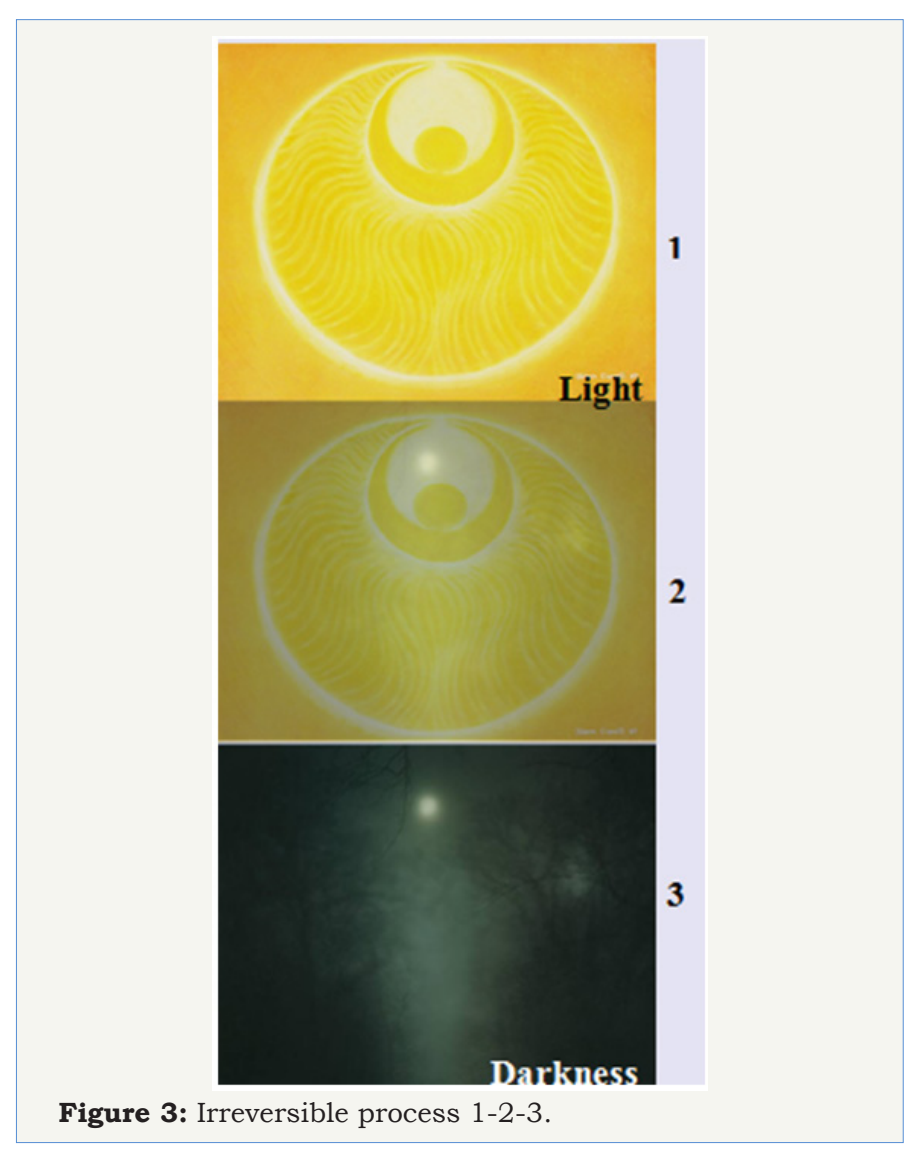

A major question is how the above transformation is achieved? In general by a thermodynamic "process" defined as "the energetic evolution of a thermodynamic system proceeding from an initial state to a final state". In other words, a system undergoes a thermodynamic process when there is some sort of energetic change within the system, generally associated with changes in pressure, volume, internal energy, temperature, or any sort of heat transfer. In principle there are two kinds of processes, a reversible and an irreversible process. A "reversible process" is defined $[8,9]$ as "a process that, once having taken place, can be reversed, and in so doing leaves no change in either the system or surroundings". In other words the system and surroundings are returned to their original condition. A reversible process of a system can take place only in infinitesimal steps about equilibrium states. Although the reversible process can be approximated, real processes can never match it. Figure 2a, "The Art of Living" (1967) painted by Rene Magritte, Belgian Surrealist painter (1898-1967), is used to demonstrate a reversible process as follows. The balloon was reconstructed 11 times and in each case its diameter was slightly reduced so that the collection 1 to 11 may demonstrate a reversible process. When a system in a given initial state goes through a number of different changes in state and finally returns to its initial values, the system has undergone a "cyclic process" or "cycle. This is demonstrated in Figure 2a by considering the steps 1-5-9-1114-18-1. An additional cyclic process is demonstrated in Figure $2 \mathrm{~b}$ by the steps 1-3-5-7-9-10. It was MC Escher [10], a Dutch graphic artist, who painted Figure 2b, entitled "Day and Night". And now the irreversible process that is the most widespread one. In this case finite changes are made; therefore the system is not at equilibrium throughout the process. Such a process is demonstrated in Figure 3 where the transformation from light to darkness is conducted in three steps that differ significantly from each other. The top, "Golden Light", and the middle artworks were painted by Jaison Cianelli (1980) an American artist where darkness was painted by the British artist Nicholas Hughes (1962-2009).

\section{Conclusion}

The authors believe that the artistic demonstrations make the subject of this article clearer and more perceptible.

\section{References}

1. What is Thermodynamics? wiseGEEK.

2. http://en.wikiquote.org/wiki/Thermodynamics

3. Andrew Zimmerman Jones (2017) Energy: A Scientific Definition

4. http://en.wikiquote.org/wiki/Arnold_Sommerfeld

5. http://images.google.co.il/imgres?imgurl=http://www.van-gogh-oncanvas.com/image

6. http://science.jrank.org/pages/6816/Thermodynamics.html

7. http://en.wikipedia.org/wiki/Thermodynamics\# Thermodynamic_ processes

8. Uvarov EB, Alan Isaacs (1993) The Penguin Dictionary of Science. ( $7^{\text {th }}$ edn), Penguin Books, UK.

9. Magritte, Harry T (1967) The True Art of Living, Abradale Press, NY, USA.

10.Escher, Locher JL (1992) The Complete Graphic Work. 